\title{
Stimulus compatibility effects of an accessory visual stimulus on auditory sensitivity
}

\author{
THOMAS F. SEIF and JAMES H. HOWARD. JR. \\ The Catholic University of America, Washington, D.C. 20017
}

\begin{abstract}
Studies have reported that an accessory stimulus in a second modality can have a facilitative effect on sensory detection in a primary modality. However, performance declines when the subject has the additional task of reporting on the accessory stimulus. The facilitative effect has been attributed to an arousal or warning process, while the interference effect has been characterized as a result of limited channel capacity. Auditory sensitivity was assessed under accessory visual stimulation. Visual stimulus intensity (arousal), processing demand, and interstimulus compatibility (same or opposite direction of change of primary and secondary stimuli) were manipulated. The results indicated that interstimulus compatibility, not arousal, accounted for the obtained facilitation effect. Interference due to additional task demands was not observed. It was proposed that the facilitation effect was due to selective sensory encoding.
\end{abstract}

Intersensory stimulation of the human auditory and visual systems has been a traditional subject of investigation (Gilbert, 1941). Generally, studies have found that simultaneous stimulation of a secondary modality facilitated the processing of sensory input in a primary modality when two conditions were met: (a) response measures to the primary stimulus were linited to threshold determination tasks (Child \& Wendt, 1938; Gregg \& Brogden, 1952; Thompson, Voss, \& Brogden, 1958; Treisman \& Howarth, 1959) or reaction time tasks (Bernstein. 1970; Bernstein \& Edelstein, 1971; Simon \& Craft. 1970), and (b) subjects were not required to respond to the secondary stimulus. ${ }^{1}$ The facilitative effect was eliminated and even reversed when the subject had to respond to the secondary stimulus as well as the primary stimulus (Lindsay, Cuddy, \& Tulving, 1965; Lindsay, Taylor. \& Forbes, 1968; Smith, 1969; Thompson et al., 1958; Tulving \& Lindsay, 1967).

These effects are consistent with Posner and Boies. (1971) tripartite model of attention. They have argued that stimuli have independent alerting. encoding, and processing properties. Thus, an accessory stimulus can serve an alerting or warning function, facilitating detection in the primary modality, while the limited channel capacity of the processing component can account for the interference effect of having to attend to the accessory stimulus.

Intersensory facilitation found in reaction time studies has typically been attributed to alerting or preparation properties of the accessory stimulus (Bernstein, 1970; Nickerson, 1973), and the likely

This paper is based on a dissertation submitted by the first author to the Catholic University of America and was supported in part by NSF Grant GU-3285 to the Catholic University and the second author. The authors acknowledge the critical comments of J. C. Townsend and F. A. Andrews. physiological mechanism involved is the reticular formation of the brainstem (Lynn. 1966; Sokolow. 1963). However, it is not clear that the alerting and processing components of the accessory stimulation are independent. Hernández-Peón (1966) has suggested that the reticular formation has descending inhibitory as well as ascending excitatory effects. If the arousal properties of the accessory stimulus are varied along with the degree to which the subject has to process the secondary stimulus, the two variables should interact.

In an unpublished master's thesis (Seif, 1969), the arousal properties of simultaneous visual stimulation were varied while auditory thresholds were determined by the method of constant stimuli. Two levels of visual stimulus intensity (zero and $27.6 \mathrm{tL}$ ) and complexity (a simple rectangular field and an equal luminance field with an enclosed randomshaped figure) were used. Both stimulus intensity and stimulus complexity directly affect arousal (Berlyne \& McDonnell. 1965; Sokolov, 1963). In addition, the subject's task load was also varied-the report group had to indicate which visual condition occurred on each trial as well as detect the tone, while the no-report group had only to detect the tone.

A trial began with a change in the visual stimulus from the intertrial level $\left(1.34 \times 10^{-2} \mathrm{fL}\right)$ to one of the three visual conditions (dark. light, or picture) which lasted for $800 \mathrm{msec}$. A $200-\mathrm{msec}$ tone burst was presented at one of seven intensities (including $0 \mathrm{~dB}$ ) $300 \mathrm{msec}$ after the beginning of the trial. All combinations of visual condition and tone intensity were presented equally often. Each of the 20 subjects per group received 315 trials.

Auditory thresholds were significantly lower with the more intense visual stimulus and lighter task load (see Figure 1). However, the greater visual stimulus 


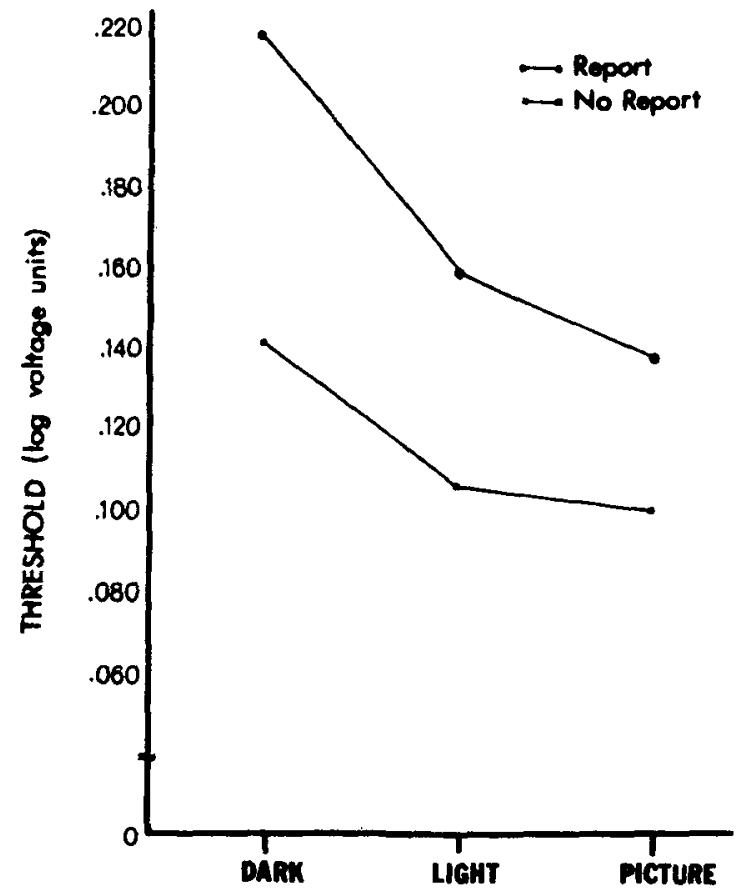

Figure 1. Auditory thresholds in log voltage units for the three visual and two task conditions.

complexity did not significantly increase sensitivity beyond that produced by the increase in brightness alone. This is consistent with the Posner and Boies (1971) conceptualization of independent arousal and processing functions, and fails to support the Hernández-Peón model.

The intensity effect observed in Seif (1969) had previously been reported for "suprathreshold" accessory stimuli in Gregg and Brogden (1952) and Thompson et al. (1958). Since all of these studies used classical threshold techniques to determine auditory sensitivity, they can be challenged for failing to adequately control variations in response bias (Green $\&$ Swets, 1966). However, Watkins (1966) used a foreed-choice procedure to determine auditory sensitivity under two accessory visual conditions, light increalse and light decrease. His results were consistent with the previous findings-auditory sensitivity was higher under light increase. Similar effects were found when visual sensitivity was measured under accessory auditory stimulation (Watkins \& Feehrer, 1965).

Although the intensity effect is consistent with the notion that performance is a direct function of arousal, there is an additional factor that can account for the facilitation observed. In each of the four studies that manipulated accessory stimulus intensity, ficilitation occurred when the direction of stimulus change was identical for both the primary and secondary stimuli (i.e.. intensity increase). Conse- quently, it may not have been the intensity level alone that produced the effect; rather, it is possible that an accessory stimulus only facilitates the detection of ipsidirectional stimuli.

Stimulus compatibility, which has been overlooked in the previous literature. fits neatly into the Posner and Boies (1971) tramework. They argued that subjects can use the information provided in a stimulus to selectively prepare for the presentation of a second stimulus. They have demonstrated convincingly that such selective preparation results in a substantial reduction in the time required to encode the second stimulus (e.g.. Kerr, 1973). If, in the Seif (1969) experiment, the prior visual stimulus provided the subjects with a perceptual "set" or model for the forthcoming auditory stimulus, then the compatible conditions should lead to a facilitation of auditory stimulus encoding, whereas the incompatible condition would not. In addition, the effect of stimulus compatibility should be independent of the effects of light energy changes that would presumably influence only the arousal component of attention.

The present experiment examines the effect of auxiliary visual stimulation on an auditory "pedestal" detection task where one group of subjects had to detect intensity increments in a continuous tone and a second group had to detect intensity decrements (cf. Green \& Swets, 1966; p. 182). Although most studies of intersensory stimulation have measured the psychophysical threshold in the classic sense, the present study involves a response-bias-free measure of sensitivity, $\mathrm{d}^{\prime}$, as elaborated in the theory of signal detectability (Green \& Swets, 1966). Earlier cross-modal studies involving this measure have reported mixed results. For example, Bothe and Marks (1970) failed to find facilitation for simultaneous bursts of light during white noise detection. On the other hand, Klayman (1973) used a three-alternative forced-choice procedure and found that a visual cue facilitated tone detection. Likewise, Watkins and Schjelderup (1967) found sensory facilitation for interstimulus intervals varying from .5 to $.2 \mathrm{sec}$, but no effect for amount of light-intensity change.

\section{METHOD}

\section{Subjects}

Eight adult subjects were randomly assigned to two groups. tonc-increment and tone-decrement detection. They were paid $\$ 12$ for participating in the $1-\mathrm{h}$ practice and four $1.5 \mathrm{~h}$ experimental se'ssions.

\footnotetext{
Apparatus

The auditory stimulus consisted of three components: a "white noise" background. a continuous $1-\mathrm{kHz}$ pedestal, and the signal. i.e. a change in the pedestal intensity. Background noise was produced by a General Radio Company 1390-A random noise generitor, while the pedestal was produced by a Hewlett Packard Model 2(1)( D wide-range oscillator. Pedestal and noise were mixed and amplitied by a simple laboratory-constructed solid-state
} 
amplitier. Signals were produced by varying the load of a photoresistor across the output of the oscillator to produce click-free tone increments and decrements (see Small, 1969. for a detailed description). Signal bursts and interstimulus intervals wete timed by three Hunter timers. Broadband $(0-20 \mathrm{kHz})$ measures of the auditory stimuli taken at the headphones (Lafayette SP-55 stereo headphones) by a General Radio $1551-\mathrm{C}$ sound-level meter were $69.5 \mathrm{~dB}\left(\right.$ re: $.0002 \mathrm{dynes} / \mathrm{cm}^{2}$ ) for the white noise and $56 \mathrm{~dB}$ for the pedestal. The pedestal and signal voltage levels were monitored throughout the experiment.

Visual stimuli were generated by a two-field Scientific Prototype Model $800 \mathrm{~F}$ tachistoscope. The tachistoscope was moditied so that the experimenter could preselect one of the four luminance levels before each trial. Luminance levels. measured by an Ilford illuminometer, were determined to be $0,2.35,16.6$, and $30.0 \mathrm{fL}$. and subtended a visual angle of $4^{\circ} 21^{\prime}$. The intertrial level was 14.16 fL. Subjects responded by pushing buttons on a panel which lit corresponding indicator lamps for the experimenter to record. Data analysis was performed on the Catholic University Computing Center's DECsystem 10 computer.

\section{Procedure}

The primary task was the detection of changes in the intensity of the pedestal in the noise background. A rating procedure was used to obtain a response-bias-free measure of auditory sensitivity (Green \& Swets, 1966). Each trial began when the subject was presented with a visual stimulus-a change in the intertrial level. Three hundred milliseconds later. a 200 -msec auditory signal burst occurred with .5 probability. The change in visual stimulus level lasted for $1 \mathrm{sec}$, after which it was replaced by the intertrial level. Subjects were instructed to respond when the intertrial level reappeared. The variable intertrial interval averaged about 5 sec.

The four levels of visual stimulus intensity consisted of light offset (O). brightness decrease (D), brightness increase to Level 1 (B1). and brightness increase to Level 2 (B2). A fitth condition (F) involved the presentation of two $10-\mathrm{msec}$ flashes at Level B2, marking the beginning and end of the $1-\mathrm{sec}$ interval. Conditions $\mathrm{F}$ and $\mathrm{B} 2$ could be compared to test the effect of absolute energy while holding relative energy change constant. Thus. there were two light-decrease conditions $(O$ and $D)$ and three light-increase conditions (B1, B2, and F).

Subjects responded by indicating, on a 6-point scale, how confident they were that an auditory signal occurred or did not occur. The ratings from 6 to 1 were described as "very confident, about $90 \%$." "moderately confident, about $70 \%$," or "slightly confident. about $55 \%$ " that a signal occurred and "slightly confident. about $55 \%$. "moderately contident about $70 \%$ " or "very contident, about $90 \%$ " that a signal did not occur. Subjects were encouraged to adjust their criteria so that they used the categories about equally often.

Subjects in the increment group were presented pedestal increments. while subjects in the decrement group were presented pedestal decrements. Signal intensities were adjusted on the first session so that the subjects correct response rate was between $70 \%$ and $80 \%$. The remaining four sessions consisted of four blocks of 120 trials. Each block was a randomization of 12 sets of the 10 visual-auditory stimulus conbinations. Briet rest periods were given between blocks.

Finally, data were collected under two task conditions, which alternated across sessions for all subjects. Task A was described above. where subjects had to report on the auditory stimuli only. Task $A-V$ included a subsequent response, indicating whether the visual stimulus on a given trial was a decrease $(O$ or $D)$ or an increase (B1. B2, or F) in brightness. Subjects were impressed with the primary nature of the auditory detection task in Condition A-V. Task order was counterbalanced across subjects.

\section{RESULTS}

Egan's dé (for convenience, $d^{\prime}$ ) was calculated under each of the $10 \mathrm{~V}$ isual by Task conditions for each subject (Green \& Swets. 1966, p. 98). These scores are response-bias-free and adjusted for unequal noise and signal-plus noise distribution variances.

Mean d's by task and visual conditions for each group are presented in Figure 2. Since the models described above do not make clear-cut predictions for the "flash" visual condition, a Lindquist Type VI analysis of variance was performed on the remaining four conditions only.

The only significant effect was the interaction of visual condition and group, $F(3,18)=3.22, p<.05$. Subjects in the increment group performed better under continuous brightness increase than under
Figure 2. Auditory sensitivity for visual stimulus, group and task condition.

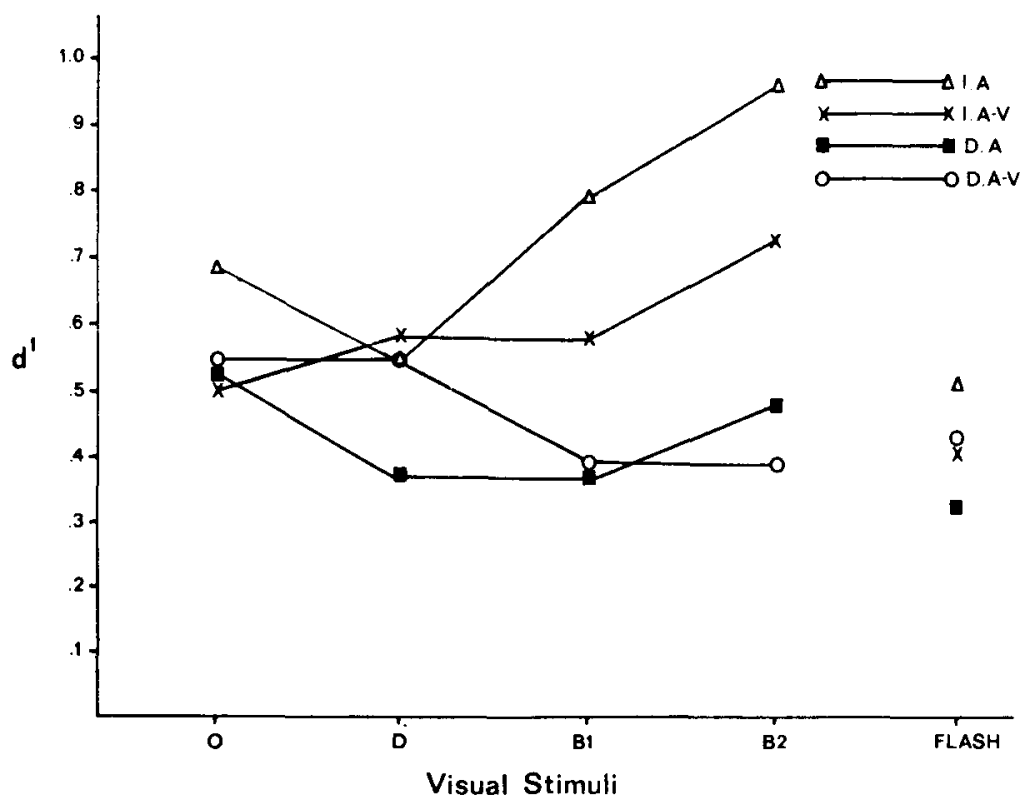




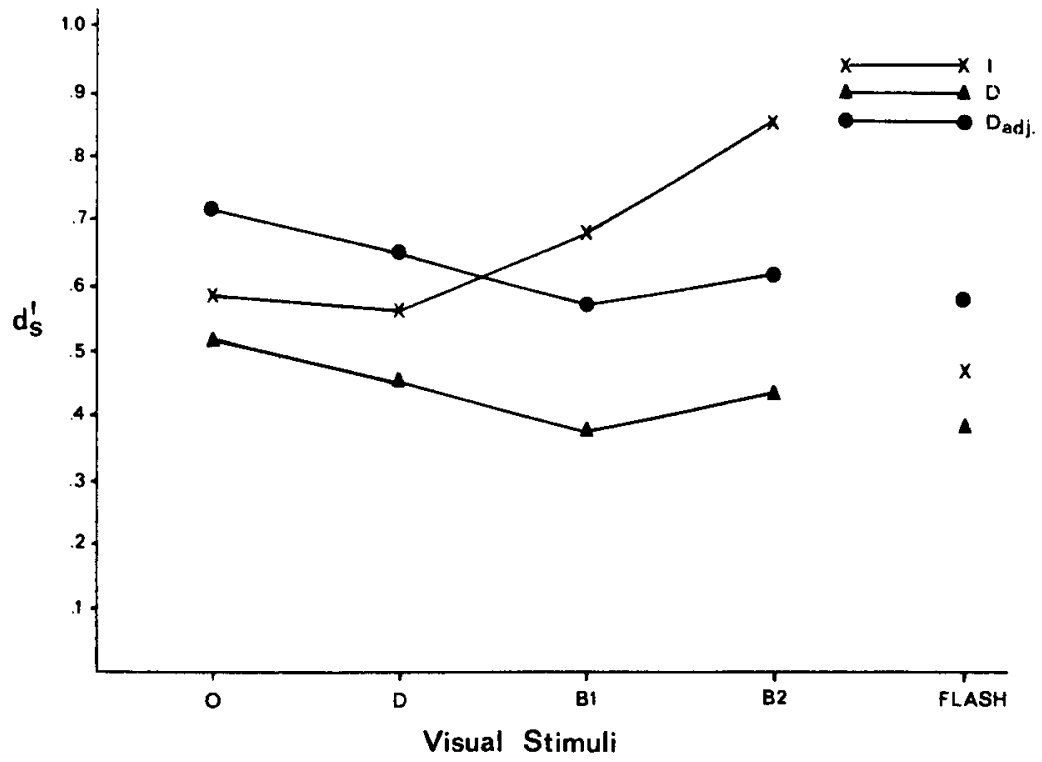

Figure 3. Auditory sensitivity by visual stimulus for Group I, D and D adjusted.

brightness decrease. The opposite effect prevailed for the decrement group.

Although the main effect for groups was not significant. the increment group performed slightly better than the decrement group. The interaction between group and visual condition is more easily seen when the data are adjusted by equating group means. The difference in mean $d^{\prime}$ for the four light conditions between the increment and decrement groups was added to each $\mathrm{d}^{\prime}$ for the decrement group. The adjusted curve is present in Figure 3.

Clearly, the significant Visual Condition by Group interaction supports the stimulus compatibility hypothesis. Neither absolute energy level nor relative energy change alone can account for the data, since the main effect for light was not significant. To determine whether these factors interact with compatibility, the eight data points were grouped into compatible and incompatible stimuli. Thus, the compatible stimuli include the increment group's visual conditions, $B 1$ and $B 2$, and the decrement group's visual conditions, $O$ and $D$. Incompatible stimuli comprise the complement set (see Figure 4).

It appears that when compatibility is controlled, $d^{\prime}$ is a positive function of energy change. However, both the compatible and incompatible regression lines have slopes which fail to differ significantly from zero, $F(1,14)=.712$ and $F(1,14)=.167$. This is likely due to the inflated values for Condition B1. One possibility is that B1 and B2 are elevated due to their greater absolute energy levels. It would be possible to examine the effect of absolute energy level with the compatibility effects removed by simply comparing the combined means for Levels $B 1$ and $B 2$ vs. $O$ and $D$

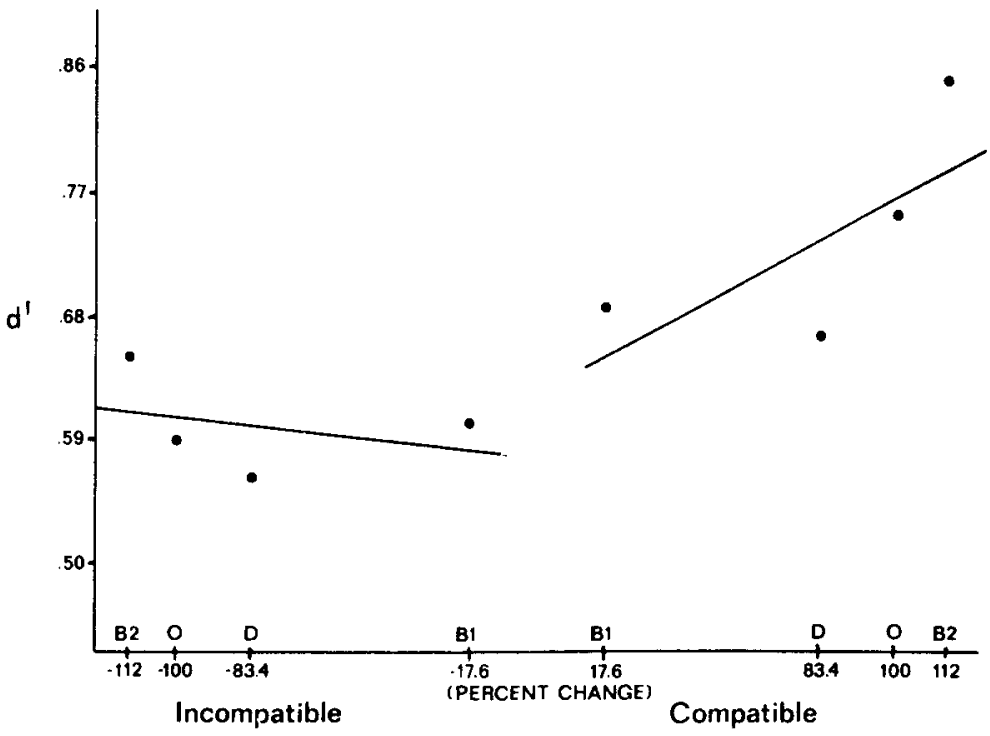

Figure 4. Auditory sensitivity by compatibility and relative energy change of visual stimuli. 
for each compatibility condition. However, the effects of relative change would still be partially confounded with the absolute energy levels. To remove the effects of relative change, the regression lines were graphically rotated about their midpoints $(\bar{x}, \bar{y})$ to the horizontal position, maintaining the relative positions of the data points. The new values were used to compute $t$ statistics for the comparisons described above. The rotation, in effect, increased the difference in means between the two bright and two dim conditions from .052 to .076 for the compatible conditions, and from .053 to .060 for the incompatible conditions. However, even this manipulation failed to produce a signiticant difference in means, $t(14)=$ $.915, p>.20$, and $t(14)=.546, p>.20$, for the compatible and incompatible conditions, respectively.

The flash condition was introduced to test two hypotheses: (a) that $d^{\prime}$ is a positive function of absolute energy $(B 2>F)$, and (b) that any detectable stimulus onset, no matter how brief, will serve as a cross-modal selective model ( $F_{\text {increm. }}>F_{\text {decrem. }}$ ). Neither hypothesis is well supported, $t(14)=1.594$, $\mathrm{p}<.10$, and $\mathrm{t}(6)=.745, \mathrm{p}>.20$, respectively. However, there appears to be an interaction between the variables, since the $\mathrm{B} 2-\mathrm{F}$ difference is significant for the increment group, $\mathrm{t}(32)=2.058, \mathrm{p}<.025$, and nonsignificant for the decrement group, $\mathrm{t}(32)=$ $0.681, p>.20$. The significant $t$ for the increment group could be interpreted as support for an absolute energy effect, but then a significant $t$ for the decrement group would also be expected. It is more likely that the $\mathrm{F}$ condition did not serve as a selective cue (viz, the significant $t$ for the increment group) and that there was no energy effect (viz, the nonsigniticant $t$ for the decrement group).

The nonsignificant task effect, $F(1,6)=0.258$, $p>.20$, suggests that the contrary findings of earlier studies were due to response bias. The task variable did not differentially affect groups or interact with light conditions.

False-alarn rates were estimated by computing the mean button response for nonsignal trials. These values are presented by group, task, and visual condition in Figure 5. An analysis of variance was performed on these data, yielding a significant visual condition main effect. $F(3,18)=6.25, p<.005$, and a significant Group by Visual Condition interaction, $F(3,18)=4.49, p<.025$. The main effect for visual condition is due to the depressed value for Condition $O$, as indicated by a least significant difference comparison.

The interaction reflects the high false-alarm rate for Condition D of the decrement group. The two groups differed significantly under Condition D only. Apparently, there is a strong negative bias for all subjects when there is a light offset. However. stimulus compatibility had a positive bias effect for the decrement group under Condition D.

\section{DISCUSSION}

The traditional concern with the arousal and warning properties of cross-modal stimulation is not sufficient to explain the data presented in the present paper. Clear support was found for a facilitative process based on ipsidirectionality. The stimulus compatibility effect is consistent with the apparently conflicting results reported in a series of studies by Watkins (ct. Seif, 1974). Detection performance was superior whenever an accessory stimulus, in the same or in a different modality, changed in the same direction as the detection stimulus (Watkins. 1964. 1966; Watkins \& Feehrer, 1965).
Figure 5. Mean button response for nonsignal trials, "bias," for visual stimulus, group and task condition.

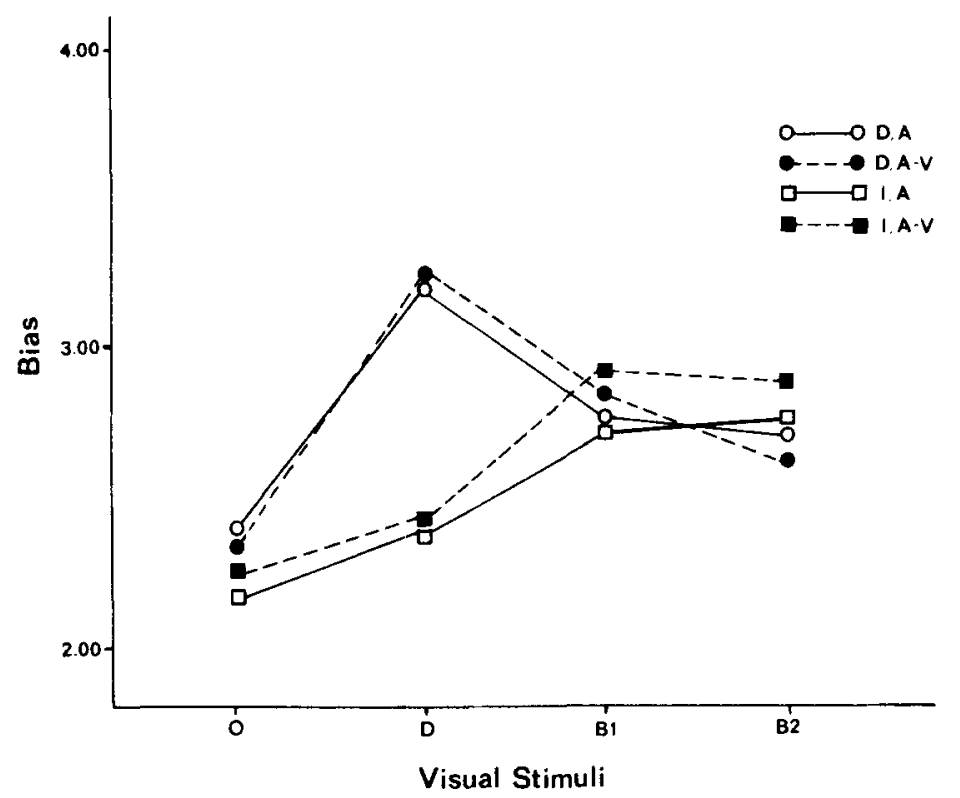


Since the compatibility effect is selective in nature, it is likely occurring at the encoding stage of processing (Posner \& Boies, 1971). A preceding stimulus establishes a "set" for relevant characteristics in an analagous way to the lowering of thresholds in attenuation models of selective attention (Morton, 1969; 'Treisman, 1964). If the subsequent stimulus is compatible with the "set." it is more easily encoded. The behavioral consequences of stimulus compatibility are increased detection and response speed.

The notion of selective encoding for nonverbal stimuli is consistent with recent work on sensory encoding. Kinchla (1973), using a "probe comparison" task, found evidence for some nonliteral, preverbal sensory storage. Likewise, Phillips (1974) claims that there is a "short-term visual memory" which is a schematic representation of the iconic image. If there is an analogous representation in the auditory system, the compatibility effect may be a result of the accessory stimulus selectively affecting the schematic representation of the primary stimulus.

Neither the interactive nor the independent effect of task and light conditions found by Thompson et al. and Seif, respectively, were replicated in the present study. In neither of the former cases, however, were pure measures of sensitivity used. The difference in tindings between Thompson et al. and Seif could be due to the near-threshold visual stimuli used by Thompson et al. as opposed to the easily detectable stimuli used by Seif (1969).

The absence of a task effect has been found in previous studies using a signal detection analysis. Broadbent and Gregory (1963) found a detrimental effect of a memory task on auditory detection only when the memory task was given primary status. A simultaneous memory task was also found to have no effect on auditory detection by Lindsay and Norman (1969). In addition, the discrimination task in the present study may have been too easy to have a negative effect-there were essentially no errors.

Lindsay and Norman (1969) argue that when there is competition between perceptual and memory tasks, it is due to a limited availability of processing capacity. This is consistent with Posner and Boies' concept of independent components of attention. The light and task variables did not interact in the present study and this supports the independence position. This was also the case for the arousal variables (relative and absolute energy). However, since main effects for the arousal and task variables were not significant, the absence of interactions may be due only to a lack of design sensitivity. The importance of stimulus compatibility in processing simple sensory stinuli, however, has clearly been demonstrated.

\section{REFERENCES}

Berlyne, D. E., \& McDonnell, P. Effects of stimulus complexity and incongruity on duration of EEG desynchronization. Electroencephalography and Clinical Neurophysiology, $1965,18,156-i 61$.

Bernstein, I. H. Can we see and hear at the same time? Acta Psychologica, 1970, 33, 21-35.

Bernstein, 1. H. \& Edelstein, A. Effects of some variation in auditory input upon visual choice reaction time. Journal of Experimental Psychology, 1971, 87, 241-247.

Bothe, G. G., \& Marks, L. E. Absolute sensitivity to white noise under auxiliary visual stimulation. Perception \& Psychophysics. 1970, 8, 176-177.

Broadbent, D. E., \& Gregory, M. Division of attention and the decision theory of signal detection. Proceedings of the Roval Society, 1963, 158, 222-231.

Child, I. L., \& WENDT, G. R. The temporal course of the influence of visual stimulation upon the auditory threshold. Journal of Experimental Psychology, 1938, 23, 109-127.

GiLbeRT, G. M. Intersensory facilitation and inhibition. Journal of General Psychology, 1941, 24, 381-407.

Green, D. M., \& Swets, J. A. Signal detection theory and psychophysics. New York: Wiley, 1966.

GregG, L. W., \& Brogden, W. J. The effect of simultaneous visual stimulation on absolute and auditory sensitivity. Journal of Experimental Psychology, 1952, 43, 179-186.

Hernández-Pé́n, R. Attention, sleep, motivation and behavior. In P. Bakan (Ed.), Attention. New York: Van Nostrand, 1966.

KERR, B. Processing demands during mental operations. Memory \& Cognition, 1973, 1, 401-412.

Kinchla, R. A. Selective processes in sensory memory: A probe-comparison procedure. In S. Kornblum (Ed.), Attention and performance IV. New York: Academic Press, 1973.

Klayman, B. Detection and the timing of a hetermodal cue: Auditory-visual interaction. Journal of Psychology, 1973, 83, 201-214.

Lindsay, P. H., Cuddy, L. L., \& Tulving, E. Absolute judgements of simultaneously presented visual and auditory stimuli. Psychonomic Science, 1965, 2, 211-212.

Lindsay, P. H., \& Norman, D. A. Short-term retention during a simultaneous detection task. Perception \& Psychophysics, 1969, 5, 201-205.

Lindsay, P. H., TAylor, M. M., \& Forbes, S. M. Attention and multidimensional discrimination. Perception \& Psychophysics, 1968, 4, 113-118.

LynN, R. Attention, arousal, and the orientation reaction. London: Pergamon Press, 1966.

Morton, J. The interaction of information in word recognition. Psychological Review, 1969, 76, 165-178.

Nickerson, R. S. Intersensory facilitation of reaction time: Energy summation or preparation enhancement? Psychological Review, 1973, 80, 489-509.

Phillips, W. A. On the distinction between sensory storage and short-term visual memory. Perception \& Psychophysics, 1974, 16. 283-290.

Posner, M., \& Boies, S. Components of attention. Psychological Review, 1971, 78, 391-408.

SEIF, T. F. The effects of visual stimulus type and subject's task on auditory sensitivity. Master's thesis. University of lowa, 1969.

SE1F, T. F. Sensory interaction: The effects of visual stimulus compatibility and task load on-auditory sensitivity. Doctoral dissertation. The Catholic University of America, 1974.

Simon, J. R., \& CRAFt, J. L. Effect of an irrelevant auditory stimulus on visual choice reaction time. Journal of Experimental Psychology, 1970, 86. 272-274. 
SMall. A. M. A simple and inexpensire click-free audio switch. Beharior Research Methods \& Instrumentation. 1969. 5. $185-18^{-}$.

Smith. M. Etfect of varying channel capacity on stimulus detection and discrimination. Journal of Experimental Ps.chology. 1909, 82, 520-520.

Sokolov. Y. N. Perception and the conditioned reflex. Vew York: Pergamon Press. 1903.

Thompson. R. F.. Voss. J. F.. \& Brogden. W. J. Effect of brightness of simultaneous visual stimulation on absolute auditory sensitivity. Joumal of Experimental Psychology. 1958. 55. 45-50.

TREISMAN. A. M. Vethal sues. language and meaning in selective attention. Americun Journal of Psichology, 1964. 77. 206-219.

TREISMas. M.. \& Howarth. C. I. Changes in threshold level produced by a signal preceding or following the threshold stimulus. Quarterly Joumal of Experimental Psychology. 1959. XI. 129-142.

Thlving. E., \& Livdsar. P. H. Identitication of simultaneously presented simple visual and auditory stimuli. Acta Psychologica. $190^{-} .27,101 \cdot 109$.
Watkins. W. H. Effect of certain noises upon detection of visual signals. Joumal of Experimental Psychology. 1964. 67. 72.75.

Watkiss. W. H. Photic facilitation of tonal signal detection in a forced-choice situation. Psychonomic Science. 1966. 6. $4^{--}-4^{-} 8$.

Watkins. W. H., \& Feehrer. C. E. Acoustic facilitation of vivual detcetion. Journal of Experimental Psychology. 1965. 70. $3.32-333$.

Watkins. W. H., \& Schjelderup. J. Effects of temporal variation of atusiliary light stimuli upon detectability of tonal signals. Perce'ption \& Psychophysics. 1967. 2. 317-322.

\section{NOTE}

1. The term "facilitation" is used in the traditional sense. Generally, studies have found better performance with accessory stimulation than without accessory stimulation (Klayman, 1973; Nickerson. 1973; Watk ins \& Schjelderup, 1967).

\section{(Received for publication October 4. 1974;} revision received December $12,1974$. 